\title{
The discontinuous solutions of Lame's equations for a conical defect
}

\author{
O. Reut, N. Vaysfeld \\ Odessa Mechnikov University, Institute of Mathematics, Economics and Mechanics, Ukraine \\ reut@onu.edu.ua,vaysfeld@onu.edu.ua
}

\begin{abstract}
In this article the discontinuous solutions of Lame's equations are constructed for the case of a conical defect. Under a defect one considers a part of a surface (mathematical cut on the surface) when passing through which function and its normal derivative have discontinuities of continuity of the first kind. A discontinuous solution of a certain differential equation in the partial derivatives is a solution that satisfies this equation throughout the region of determining an unknown function, with the exception of the defect points. To construct such a solution the method of integral transformations is used with a generalized scheme. Here this approach is applied to construct the discontinuous solution of Helmholtz's equation for a conical defect. On the base of it the discontinuous solutions of Lame's equations are derived for a case of steady state loading of a medium.
\end{abstract}

KEYwORDS. Conical defect; Helmholtz's equation; Wave potential; Integral Transformation; Lame's equations.

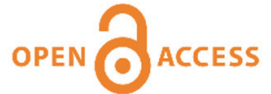

Citation: Reut, O., Vaysfeld, N., The discontinuous solutions of Lame's equations for a conical defect, Frattura ed Integrità Strutturale, 45 (2018) 183-190.

Received: 15.05 .2018

Accepted: 24.06.2018

Published: 01.07.2018

Copyright: (C) 2018 This is an open access article under the terms of the CC-BY 4.0, which permits unrestricted use, distribution, and reproduction in any medium, provided the original author and source are credited.

\section{INTRODUCTION}

$\mathrm{T}$ he urgency of the problem of elastic waves diffraction is due to the need to take into account the presence of heterogeneities during the development of new composite materials, geophysical and seismological studies. These processes take place under dynamic loads of different nature. In order to facilitate engineering design, preliminary calculations are required on the basis of appropriate mathematical models that provide an opportunity to analyze the effects of such dynamic stress concentrators as inclusions, cavities, cracks, holes, etc.

On the other hand, the problems of elastic wave diffraction are one of the classical problems of the mechanics of deformable bodies. The construction of their analytical solutions, analysis of wave fields in the vicinity of $t$ defects constitute a broad class of problems whose decompositions require the involvement of complex mathematical apparatus.

The development of this mathematical apparatus has been carried out by many scientists [1-14]. One of the powerful methods for solving problems of wave diffraction on defects of various forms is the method of discontinuous solutions.

This method was created by G. Ya. Popov [15]. He gave a definition of a discontinuous solution of the differential equation in partial derivatives, namely: a discontinuous solution of a certain differential equation in partial derivatives is such a solution that satisfies this equation throughout the region of determining an unknown function, with the exception of the defect points, in the transition through which an unknown function has discontinuities with jumps of the unknown function itself and its normal derivative. 
The jumps of the function and its normal derivative are given by the conditions of the original problem, both are established under certain conditions of the problem solving. Under a defect one must understand a part of a surface (mathematical cut on the surface) when passing through which function and its normal derivative have discontinuities of continuity of the first kind. G.Ya. Popov proposed a method for constructing such solutions for defects and bodies, described in the orthogonal curvilinear coordinate systems. It is essential, corresponding to this method, to construct a discontinuous solution of a Helmholtz's equation (or the Laplace equation in a static statement of a problem) and to further construct discontinuous solutions of the equations of motion (or equilibrium equations respectively). It is possible to realize this thanks to formulas connecting the wave potentials with the displacements and stress. In [15] the solutions of elasticity static problems were constructed for linear, circular, spherical and cylindrical defects. The method of discontinuous solutions was extended to the problem of wave diffraction in papers [16-18]. In [19] the method of discontinuous solutions is extended to the defect of an arbitrary form.

The novelty of the proposed paper is in the construction of the discontinuous solutions of Lame's equations for a conical defect in the case of a steady state loading. As a first stage of the solution deriving, a discontinuous solution of the Helmholtz equation for a case of a conical shape defect is constructed. The special scheme is proposed to find the unknown jumps of the displacements and stress. The derived formulae of Lame's equations discontinuous solutions can be applied for the solving of the boundary problems of elasticity for the different types of the conical defects, such as a crack, a thin inclusion adherent with a medium, partially adherent inclusion etc. For the case of an axisymmetric problem for a conical defect all obtained formulae are substantially simplified.

\section{STATEMENT OF THE PROBLEM}

$\mathrm{L}$

et's consider an acoustic medium containing a conical defect whose surface is described in a spherical coordinate system by the correspondences:

$$
a<r<b, 0<\theta<\omega,-\pi<\varphi<\pi
$$

The steady-state oscillations of the media are described by the Helmholtz equation

$$
\left(r^{2} \tilde{\Phi}^{\prime}(r, \theta, \varphi)\right)^{\prime}-\nabla \tilde{\Phi}(r, \theta, \varphi)-r^{2}\left(\frac{i \omega}{c}\right)^{2} \tilde{\Phi}(r, \theta, \varphi)=0
$$

here $\Phi(r, \theta, \varphi, t)=\tilde{\Phi}(r, \theta, \varphi) e^{i \omega t}, c$ is the wave's speed in the acoustic medium. Here it was agreed to disregard the designation "tilda" over a letter and to introduce the following new notations $q^{2}=\left(\frac{i \omega}{c}\right)^{2}=-\frac{\omega^{2}}{c^{2}}$, where $\omega$ is the frequency of the incident wave, $\nabla \Phi(r, \theta, \varphi)=\frac{\partial \Phi}{\partial \sin ^{2} \theta}-\frac{\left[\sin \theta \Phi^{\bullet}(r, \theta, \varphi)\right]^{\bullet}}{\sin ^{2} \theta}$, (here the point over the letter denotes a derivative with regard to a second variable).

The aim is the deriving of the discontinuous solution of the Eqn. (2) for the defect (1) located in acoustic medium.

\section{DERIVING OF THE DISCONTINUOUS SOLUTION IN THE TRANSFORMATION DOMAIN}

$\mathrm{T}$ he integral Fourier transformation is applied to the equation with regard of variable $\varphi$

$$
\Phi_{n}(r, \theta)=\int_{-\pi}^{\pi} e^{i n \varphi} \Phi(r, \theta, \varphi) d \varphi
$$

In the transformation's domain (3) the Eqn. (2) takes a form 


$$
\left(r^{2} \Phi_{n}{ }^{\prime}(r, \theta)\right)^{\prime}-\nabla_{n} \Phi_{n}(r, \theta)-r^{2} q^{2} \Phi_{n}(r, \theta)=0 \quad \nabla_{n} \Phi_{n}(r, \theta)=\frac{n^{2}}{\sin ^{2} \theta}-\frac{\left[\sin \theta \Phi_{n}^{\bullet}(r, \theta)\right]^{\bullet}}{\sin ^{2} \theta}
$$

A change of variables $r=\frac{x}{q}$ was done in the Eqn. (4). It was rewritten with the new variables in the following form

$$
\left(x^{2} \Phi_{n}^{\prime}\left(\frac{x}{q}, \theta\right)\right) \cdot-\nabla_{n} \Phi_{n}\left(\frac{x}{q}, \theta\right)-x^{2} \Phi_{n}\left(\frac{x}{q}, \theta\right)=0
$$

The Kantorovich-Lebedev integral transformation with regard to variable $x$ is applied to the equality (5)

$$
\Phi_{n \tau}(\theta)=\int_{0}^{\infty} \frac{K_{i \tau}(x)}{\sqrt{x}} \Phi_{n}\left(\frac{x}{q}, \theta\right) d x
$$

In the transformations (6) domain the Eqn. (5) can be reformulated as

$$
\tau^{2} \Phi_{n \tau}(\theta)+\left(\frac{1}{4}+\nabla_{n}\right) \Phi_{n \tau}(\theta)=0
$$

There is no possibility to apply the integral Legendre transformation by the usual scheme to the Eqn. (7) because there are discontinuities of the function $\Phi_{n \tau}(\theta)$ and its derivative when $\theta=\omega$. The jumps have the following form

$$
\begin{aligned}
& \left\langle\Phi\left(\frac{x}{q}, \theta, \varphi\right)\right\rangle=\Phi\left(\frac{x}{q}, \omega-0, \varphi\right)-\Phi\left(\frac{x}{q}, \omega+0, \varphi\right) \\
& \left\langle\Phi^{\bullet}\left(\frac{x}{q}, \omega, \varphi\right)\right\rangle=\left.\frac{\partial \Phi\left(\frac{x}{q}, \theta, \varphi\right)}{\partial \theta}\right|_{\theta=\omega-0}-\left.\frac{\partial \Phi\left(\frac{x}{q}, \theta, \varphi\right)}{\partial \theta}\right|_{\theta=\omega+0}
\end{aligned}
$$

The integral Legendre's transformation is applied to the Eqn. (7) by the generalized scheme [15]

$$
\Phi_{n \tau k}=\int_{0}^{\pi} \Phi_{n \tau}(\theta) P_{k}^{|n|}(\cos \theta) \sin \theta d \theta
$$

It leads to the linear algebraic equation in the transformations (3), (6), (9) domain

$$
\Phi_{n \tau k}\left(\tau^{2}+(k+1 / 2)^{2}\right)=\sin \omega\left(\left\langle\Phi_{n \tau}^{\cdot}(\omega)\right\rangle P_{k}^{|n|}(\cos \omega)-\left.\left\langle\Phi_{n \tau}(\omega)\right\rangle \frac{d P_{k}^{|n|}(\cos \theta)}{d \theta}\right|_{\theta=\omega}\right)
$$

We will accept the designation $\left.\frac{d P_{k}^{|n|}(\cos \theta)}{d \theta}\right|_{\theta=\omega}=\frac{d P_{k}^{|n|}(\cos \omega)}{d \omega}$ in future. Here 


$$
\left(\begin{array}{c}
\left\langle\Phi_{n \tau}(\omega)\right\rangle \\
\left\langle\Phi_{n \tau}^{\bullet}(\omega)\right\rangle
\end{array}\right)=\int_{-\pi}^{\pi} \int_{0}^{\infty} \frac{K_{i \tau}(x)}{\sqrt{x}} e^{i n \varphi}\left(\begin{array}{l}
\left\langle\Phi\left(\frac{x}{q}, \omega, \varphi\right)\right\rangle \\
\left\langle\Phi \cdot\left(\frac{x}{q}, \omega, \varphi\right)\right\rangle
\end{array}\right) d \varphi d x
$$

Finally, we derive the expression for the function's transformation $\Phi_{n \tau k}$ through the transformations of its jump and the jump of its normal derivative

$$
\Phi_{n \tau k}=\frac{\sin \omega\left(\left\langle\Phi_{n \tau}^{\bullet}(\omega)\right\rangle P_{k}^{|n|}(\cos \omega)-\left\langle\Phi_{n \tau}(\omega)\right\rangle P_{k}^{|n|}(\cos \omega)\right)}{t^{2}+(k+1 / 2)^{2}}
$$

\section{DERIVING THE FINAL FORMULA}

he inverse Legendre's transformation is applied to (10)

$$
\Phi_{n \tau}(\theta)=\sum_{k=|n|}^{\infty} \sigma_{k n} P_{k}^{|n|}(\cos \theta) \Phi_{n \tau k}
$$

where $\sigma_{k n}=\frac{(k+1 / 2)(k-|n|) !}{(k+|n|) !}$

Then the inverse Kantorovich-Lebedev transformation is applied to the obtained expression

$$
\Phi_{n}\left(\frac{x}{q}, \theta\right)=\int_{0}^{\infty} \tau s h(\pi \tau) \frac{K_{i \tau}(x)}{\sqrt{x}} \Phi_{n \tau}(\theta) d \tau
$$

Bearing in mind that the expressions for the transformations of the wave potential jumps and its normal derivative have the following form

$$
\left[\begin{array}{c}
\left\langle\Phi_{n \tau}(\omega)\right\rangle \\
\left\langle\Phi_{n \tau}^{\cdot}(\omega)\right\rangle
\end{array}\right]=\int_{0}^{\infty}\left[\begin{array}{l}
\left\langle\Phi_{n}\left(\frac{\xi}{q}, \omega\right)\right\rangle \\
\left\langle\Phi_{n}^{\cdot}\left(\frac{\xi}{q}, \omega\right)\right\rangle
\end{array}\right] \frac{K_{i \tau}(\xi)}{\sqrt{\xi}} d \xi
$$

in the Fourier's transformation domain, the wave potential has the following form

$$
\begin{aligned}
& \Phi_{n}\left(\frac{x}{q}, \theta\right)=\sin \omega \sum_{k=|n|}^{\infty} \sigma_{k n} P_{k}^{|n|}(\cos \theta) \iint_{0}^{\infty} \int_{0}^{\infty} \frac{\tau s h(\pi \tau)}{\left[\tau^{2}+(k+1 / 2)^{2}\right]} \frac{K_{i \tau}(x)}{\sqrt{x}} \frac{K_{i \tau}(\xi)}{\sqrt{\xi}} . \\
& {\left[P_{k}^{|n|}(\cos \omega)\left\langle\Phi_{n}^{\bullet}\left(\frac{\xi}{q}, \omega\right)\right\rangle-P_{k}^{|n| \bullet}(\cos \omega)\left\langle\Phi_{n}\left(\frac{\xi}{q}, \omega\right)\right\rangle\right] d \xi d \tau}
\end{aligned}
$$

The integral in the last formula is known [2.16.52(11), 20] 


$$
J_{k}(x, \xi)=\frac{2}{\pi^{2}} \int_{0}^{\infty} \frac{\tau \operatorname{sh}(\pi \tau) K_{i \tau}(x) K_{i \tau}(\xi)}{\left[\tau^{2}+(k+1 / 2)^{2}\right]} d \tau=\frac{\pi i}{2 \sqrt{x \xi}}\left\{\begin{array}{c}
J_{v}(\xi q) H_{v}^{1}(x q), \xi<x \\
J_{v}(x q) H_{v}^{1}(\xi q), \xi>x
\end{array}, v=k+1 / 2\right.
$$

The discontinuous solution can be simplified

$$
\Phi_{n}\left(\frac{x}{q}, \theta\right)=\sin \omega \int_{0}^{\infty}\left(\left\langle\Phi_{n}^{\cdot}\left(\frac{\xi}{q}, \omega\right)\right\rangle G_{n}(x, \xi ; \theta, \omega)-\left\langle\Phi_{n}\left(\frac{\xi}{q}, \omega\right)\right\rangle \frac{\partial G_{n}(x, \xi ; \theta, \omega)}{\partial \omega}\right) d \xi, x=r q, \xi=\rho q
$$

here $G_{n}(x, \xi ; \theta, \omega)=\sum_{k=|n|}^{\infty} \sigma_{k n} P_{k}^{|n|}(\cos \theta) P_{k}^{|n|}(\cos \omega) J_{k}(x, \xi)$.

It was stated that the limit values of the wave potential near the branches of the defect (1) have the form

$$
\begin{aligned}
& \Phi_{n}\left(\frac{x}{q}, \omega \mp 0\right)= \pm \frac{1}{2}\left\langle\Phi_{n}\left(\frac{x}{q}, \omega\right)\right\rangle- \\
& -\sin \omega \int_{0}^{\infty}\left(\left.\left\langle\Phi_{n}\left(\frac{\xi}{q}, \omega\right)\right\rangle \frac{\partial G_{n}(x, \xi ; \theta, \omega)}{\partial \omega}\right|_{\theta=\omega \mp 0}-\left.\left\langle\Phi_{n}^{\cdot}\left(\frac{\xi}{q}, \omega\right)\right\rangle G_{n}(x, \xi ; \theta, \omega)\right|_{\theta=\omega \mp 0}\right) d \xi, x=r q, \xi=\rho q
\end{aligned}
$$

These formulas are derived by the use of the known facts of potential theory such as a discontinuity of a double layer's potential and normal derivative of plane layer potential.

The application of inverse integral Fourier's transformation to formula (12) completes the construction of discontinuous solution of Helmholtz's equation.

\section{CONSTRUCTION OF THE DISCONTINUOUS SOLUTIONS OF LAME’S EQUATIONS}

A ccordingly to the discontinuous solution method [15] to derive the discontinuous solutions of Lame's equations, one must find the formulae expressing the jumps of the wave potentials and their derivatives through the jumps of the displacements and stress. The wave potential functions $\Phi(r, \theta, \varphi), \Psi_{j}(r, \theta, \varphi), j=1,2$ satisfy the Helmholtz's Eqns. (2) with the velocities $c_{1}, c_{2}$ correspondently.

Well known formulas in the Fourier's transformation domain (3) are written in the form [11]:

$$
\begin{aligned}
& u_{n}(r, \theta)=\Phi_{n}^{\prime}(r, \theta)+r^{-1} \nabla_{n} \Psi_{2 n}(r, \theta), \\
& v_{n}(r, \theta)=r^{-1}\left(\Phi_{n}^{\bullet}(r, \theta)+\left(r \Psi_{2 n}^{\bullet}(r, \theta)\right)^{\prime}\right)+i n \sin ^{-1}(\theta) \Psi_{1 n}(r, \theta), \\
& w_{n}(r, \theta)=i n(r \sin \theta)^{-1}\left(\Phi_{n}(r, \theta)+\left(r \Psi_{2 n}(r, \theta)\right)^{\prime}\right)-\Psi_{1 n}^{\bullet}(r, \theta),
\end{aligned}
$$

here $U_{r}^{n}(r, \theta)=u_{n}(r, \theta), U_{\theta}^{n}(r, \theta)=v_{n}(r, \theta), U_{\varphi}^{n}(r, \theta)=w_{n}(r, \theta)$. The stress transformations are expressed through the displacements and hence through the wave potentials as well.

To use the discontinuous solution of the Helmholtz's Eqn. (2) derived earlier, the jumps' transformations of the wave functions $\Phi_{n}(r, \theta), \Psi_{j n}(r, \theta), j=1,2$ should be expressed through the jumps of the stress and displacements. This procedure is enough complicated, so here its scheme is shown.

One must use such equalities $\nabla_{n} \Psi_{2 n}(r, \theta)=L_{c_{2}} \Psi_{2 n}(r, \theta), \nabla_{n} \Phi_{n}(r, \theta)=L_{c_{1}} \Phi_{n}(r, \theta), L_{c} y=\left(r^{2} y^{\prime}\right)^{\prime}+r^{2} \omega^{2} c^{-2}$. With regard of these formulas, it is possible to express the jumps of the mechanical characteristic through the jumps of the wave potentials: 


$$
\begin{aligned}
& \left\langle u_{n}(r, \omega)\right\rangle=\left\langle\Phi_{n}^{\prime}(r, \omega)\right\rangle+r^{-1} L_{c_{2}}\left\langle\Psi_{2 n}(r, \omega)\right\rangle \\
& \left\langle v_{n}(r, \omega)\right\rangle=r^{-1}\left(\left\langle\Phi_{n}^{\bullet}(r, \omega)\right\rangle+\left(r\left\langle\Psi_{{ }_{2 n}}(r, \omega)\right\rangle\right)^{\prime}\right)+i n \sin ^{-1} \omega\left\langle\Psi_{1 n}(r, \omega)\right\rangle \\
& \left\langle w_{n}(r, \omega)\right\rangle=i n(r \sin \omega)^{-1}\left(\left\langle\Phi_{n}(r, \omega)\right\rangle+\left(r\left\langle\Psi_{2 n}(r, \omega)\right\rangle\right)^{\prime}\right)-\left\langle\Psi_{1 n}^{\bullet}(r, \omega)\right\rangle \\
& \left\langle\sigma_{\theta n}(r, \omega)\right\rangle=2 G\left(q^{2} \mu_{*}\left\langle\Phi_{n}(r, \omega)\right\rangle+r^{-1}\left(\left\langle v_{n}^{\bullet}(r, \omega)\right\rangle-\left\langle u_{n}(r, \omega)\right\rangle\right)\right), \\
& \left\langle\tau_{r n}(r, \omega)\right\rangle=G\left(r\left(r^{-1}\left\langle v_{n}(r, \omega)\right\rangle\right)^{\prime}+r^{-1}\left\langle\dot{u}_{n}(r, \omega)\right\rangle\right), \\
& r\left\langle\tau_{\varphi_{n}}(r, \omega)\right\rangle=G\left(\left\langle w^{\bullet}(r, \omega)\right\rangle-\operatorname{ctg} w_{n}\left\langle w_{n}(r, \omega)\right\rangle+i n \operatorname{cosec} \omega\left\langle v_{n}(r, \omega)\right\rangle\right)
\end{aligned}
$$

In the formulae (14) the jump $\left\langle v_{n}^{\bullet}(r, \omega)\right\rangle$ should be excluded. The volume expansion's transformation $\Theta_{n}(r, \theta)$ is expressed through the displacements transformations to realize it. It is proved that the equality $\Theta_{n}(r, \theta)=q^{2} \Phi_{n}(r, \theta)$ is true also. As a result, the jump $\left\langle v_{n}{ }_{n}(r, \omega)\right\rangle$ is derived

$$
\left\langle v_{n}{ }_{n}(r, \omega)\right\rangle=r q^{2}\left\langle\Phi_{n}(r, \omega)\right\rangle-r^{-1}\left(r^{2}\left\langle u_{n}(r, \omega)\right\rangle\right)^{\prime}-\operatorname{ctg} \omega\left\langle v_{n}(r, \omega)\right\rangle-i n \sin ^{-1} \omega\left\langle w_{n}(r, \omega)\right\rangle
$$

Hence, from the formula for the normal stress jump (14), the jump of the scalar wave potential is expressed only through the given jumps of the stress and displacements

$$
\mu_{0}\left\langle\Phi_{n}(r, \omega)\right\rangle=(2 G)^{-1}\left\langle\sigma_{\theta n}(r, \omega)\right\rangle+r^{-1} \operatorname{ctg} \omega\left\langle v_{n}(r, \omega)\right\rangle+r^{-1}\left(r\left\langle u_{n}(r, \omega)\right\rangle\right)^{\prime}+i n(r \sin \omega)^{-1}\left\langle w_{n}(r, \omega)\right\rangle
$$

The formula is constructed for the jump $\left\langle\Psi_{1 n}(r, \omega)\right\rangle$ where it is expressed through the jumps of the displacements and stress only

$$
L_{c_{2}}\left\langle\Psi_{1 n}(r, \omega)\right\rangle=G^{-1} r\left\langle\tau_{\varphi n}(r, \omega)\right\rangle-2 \operatorname{in}(\sin \omega)^{-1}\left\langle v_{n}(r, \omega)\right\rangle+2 \operatorname{ctg} \omega\left\langle w_{n}(r, \omega)\right\rangle .
$$

As it seen this formula is the differential equation with regard of unknown jump $\left\langle\Psi_{1 n}(r, \omega)\right\rangle$. To solve this equation a change of variable $r=\frac{x}{q}$ was done for Eqn. (15) and integral transformation (6) was applied to both part of the equation. The unknown jump was written in the transformation' domain

$$
\begin{aligned}
& \left\langle\Psi_{1 n \tau}(\omega)\right\rangle=-\frac{1}{\frac{1}{4}+\tau^{2}}\left[(G s)^{-1} \int_{0}^{\infty} \frac{\xi\left\langle\tau_{\varphi n}\left(\frac{\xi}{s}, \omega\right)\right\rangle}{\sqrt{\xi}} K_{i \tau}(\xi) d \xi+2 \operatorname{ctg} \omega \int_{0}^{\infty} \frac{\left\langle w_{n}(r, \omega)\right\rangle}{\sqrt{\xi}} K_{i \tau}(\xi) d \xi+\right. \\
& \left.-2 \operatorname{in}(\sin \omega)^{-1} \int_{0}^{\infty} \frac{\left\langle v_{n}(r, \omega)\right\rangle}{\sqrt{\xi}} K_{i \tau}(\xi) d \xi\right]
\end{aligned}
$$

The inverse integral Kantorovich-Lebedev transformation is applied to the obtained expression (16), and final solution is derived in the form 
$\left\langle\Psi_{1 n}(r, \omega)\right\rangle=-s \int_{0}^{\infty}\left[s \frac{\left\langle\tau_{\varphi n}\left(\frac{\xi}{s}, \omega\right)\right\rangle}{G}+2 \operatorname{ctg} \omega\left\langle w_{n}(r, \omega)\right\rangle+2 \operatorname{in}(\sin \omega)^{-1}\left\langle v_{n}(r, \omega)\right\rangle J_{k}(r s, \rho s) d \rho, \quad s=-\omega^{2} c_{2}^{-2}\right.$

The jump of wave function $\left\langle\Psi_{2 n}(r, \omega)\right\rangle$ is found by the analogical procedure

$$
\left\langle\Psi_{2 n}(r, \omega)\right\rangle=s \int_{0}^{\infty} \rho\left(\left\langle\Phi_{n}^{\bullet}(r, \omega)\right\rangle^{\prime}-\left\langle u_{n}(r, \omega\rangle\right) J_{k}(r s, \rho s) d \rho\right.
$$

The jumps of the wave functions derivatives are constructed using this scheme. For example, the expression for the derivative of wave potential jump $\left\langle\Psi_{2 n}(r, \omega)\right\rangle$ has the form

$$
\left\langle\Psi_{2 n}^{\bullet}(r, \omega)\right\rangle=r \int_{0}^{r} \rho\left(\left\langle v_{n}(\rho, \omega)\right\rangle^{\prime}-i n(\sin \omega)^{-1}\left\langle\Psi_{1 n}(\rho, \omega)\right\rangle\right) d \rho
$$

As a result, all transformations of the wave functions and their derivatives jumps are expressed through the transformations of stress and displacements jumps. There are substituted to the corresponded formulae of the Helmholtz's equation discontinuous solution and inversion of the integrals transformations is done. The following substitution of these formulae to the expressions (13) finalizes the deriving of the discontinuous solutions of Lame's equations in a case of steady state oscillations for the defect (1).

\section{CONCLUSIONS}

1. The discontinuous solution of Helmholtz's equation is derived for a conical defect.

2. To construct the discontinuous solutions of Lame's equations for a conical defect one must express the jumps of wave functions and their derivatives through the displacements and stress jumps. The substitution of these formulas to the discontinuous solution of Helmholtz's equation leads to the discontinuous solutions of the Lame's equations.

3. In the case when a defect is a crack, the jumps of the stress are equal to zero, when a defect is a thin shell adherent to a medium the jumps of the displacements are equal to zero. So, the derived formulae can be used for the different types of the conical defects situated in a medium.

4. The derived formulae will be significantly simplified when the axisymmetrical problem is solved. In this case parameter $n$ should be equal to zero in all final expressions.

\section{ACKNOWLEDGMENTS}

he authors are grateful to Simon Peter Dyke for his attention and great help in the editing of the manuscript's text.

\section{REFERENCES}

[1] Babeshko, V. A., Babeshko, O. M. and Evdokimova, O. V. (2010). On the method of block element, Mechanics of Solids, 45, pp. 437-444. DOI: 10.3103/S0025654410030143.

[2] Grinchenko, V. T. and Meleshko, V. V. (1981). Harmonical oscillations and waves in elastic bodies (in Russian), Naukova dumka, Kyiv. 
[3] Guz, A. N., Kubenko, V. D. and Cherevko, M. A. (1978). Diffraction of elastic waves(in Russian), Naukova dumka, Kyiv.

[4] Mykhas'kiv, V., Stankevych, V., Zhbadynskyi, I. and Zhang, Ch. (2009). 3-D dynamic interaction between a pennyshaped crack and a thin interlayer joining two elastic half-spaces, International Journal of Fracture, 159(2), pp. 137-149.

[5] Slepyan, L. I., Mechanics of cracks (in Russian), Sudostroenie, Leningrad (1990).

[6] Mnev, E. N. and Perzev, A. K. (1970). Hydroelasticity of shells (in Russian), Sudostroenie, Leningrad.

[7] Vilde, M. V., Kaplunov, Yu. D. and Kossovich, L. Yu. (2010). Boundary and interfacial resonance effects in elasticity bodies (in Russian), Fizmatgiz, Moscow.

[8] Kit, G. S. and Khay, M. V. (1989). Method of potentials in three-dimensional problems for thermoelasticity bodies with cracks (in Russian), Naukova dumka, Kyiv.

[9] Sladek, V. and Sladek, J. (1984). Transient elastodynamic three-dimensional problems in cracked bodies/Applied Mathematical Modelling, 8(1), pp. 2-10.

[10] Guz, A.N., Guz, I.A., Men'shikov, A.V. and Men'shikov, V.A. (2011). Stress-intensity factors for materials with interface cracks under harmonic loading, Int Appl Mech, 46, pp. 1093. DOI: 10.1007/s10778-011-0401-1.

[11] Savruk, M. P., Osiv, P. N. and Prokopchuk, I. V. (1989). Numerical analysis in plane problems of the crack's theory (in Russian), Naukova dumka, Kyiv.

[12] Di Cocco, V. and Iacoviello, F. (2017). Ductile cast irons: Microstructure influence on the damaging micromechanisms in overloaded fatigue cracks, Engineering Failure Analysis, 82, pp. 340-349.

[13] Toribio, J., Gonzàles, B. and Matos, J.C. (2017). Crack tip field in circumferentially-cracked round bar (CCRB) in tension affected by loss of axial symmetry, Frattura ed Integrità Strutturale, 41, pp. 139-142. DOI: 10.3221/IGF-ESIS.41.19.

[14] Peron, M., Razavi, S.M.J., Berto, F. and Torgersen, J. (2017). Notch stress intensity factors under mixed mode loadings: an overview of recent advanced methods for rapid calculation, Frattura ed Integrità Strutturale, 42, pp. 196-204. DOI: 10.3221/IGF-ESIS.42.21.

[15] Popov, G.Ya. (1982). The elastic stress' concentration around dies, cuts, thin inclusions and reinforcements (in Russian), Nauka, Moskow.

[16] Popov, V. G., (1995). The vertical oscillations of a boundary hard inclusion under harmonic loading, Applied Mechanics, 76(31), pp. 46-54.

[17] Vaisfel'd, N.D., (2005). Time-dependent problems of the concentration of elastic stresses near a conical defect, Journal of Applied Mathematics and Mechanics, 69(3), pp. 427-437.

[18] Vaisfel'd, N.D. and Popov, G.Ya., (2001). The stress concentration around a semi-infinite cylindrical crack during the shock loading of an elastic medium by a centre of rotation, Journal of Applied Mathematics and Mechanics, 65(3), pp. 509-518.

[19] Reut, V. V., Fesenko, H. O., Vaysfel'd, N. and Zhuravlova, Z., (2017). Orthogonal polynomials method and its generalization at some new problems of fracture mechanics/ June 2017, Conference 14-th Intern. Conference on Fracture (ICF 14), Rhodes, Greece.

[20] Prudnikov, A.P., Brychkov, Yu. A. and Marichev, O. M., (1984). Integrals and series: Special functions. M.: Nauka (in Russian). 